\title{
Organic Monolayer Determination on Semiconductor Substrates
}

\author{
Douglas J. Guerrero, Joyce Lowes, Ramil Mercado \\ Brewer Science, Inc. \\ Rolla, MO 65401, USA \\ dguerrero@brewerscience.com
}

\begin{abstract}
Organic materials are routinely coated on semiconductor substrates for various photolithographic processes. The cleanliness of a substrate after being coated with a bottom anti-reflective coating (BARC), photoresist, or both was studied using contact angle measurement, atomic force microscopy (AFM), and ellipsometry. The relationship between the various methods was correlated. It was found that all organic materials leave a monolayer of residue. These materials have higher affinity to silicon than for silicon oxide substrates.
\end{abstract}

Keywords: monolayer, photoresist, contact angle, residue

\section{Introduction}

Organic materials are commonly used in the photolithographic process. Typically, photoresists and BARC formulations are composed of polymers and smaller molecules such as amine quenchers, photoacid or thermal acid generators, surfactants, crosslinkers, and other additives. After processing, it is always desirable that these materials be completely removed from the substrate. However, in reality, strong adhesion forces between materials and substrates can keep a small amount, or a monolayer, of residue on the substrate surface. This small amount of material is so tightly bound to the substrate that washing with organic solvents or aqueous solutions are not able to remove them from the surface. Often, these monolayers are a few angstroms thick and are not visible using standard SEM examination techniques.

Polymer adhesive forces at interfaces have been extensively studied in the past ${ }^{1,2}$. It has been shown that the role of surface energies and hydrogen bonds play a significant role in material adhesion to substrates $^{3}$. In this paper we wanted to quantify the amount of residue left on a silicon substrate and determine the best method for measurement. We used contact angle measurement, ellipsometry, and atomic force microscopy to measure monolayer residue after photoresist processing with and without using a developable BARC. Finally, we compare the various methods in their ability to detect monolayer residues.

\section{Method}

2.1 Materials and process

Several commercially available ArF photoresists were tested with and without an organic photosensitive BARC. The photoresists were processed using the manufacturer-recommended thickness and bake conditions. The resist was coated on 8-inch silicon or BARC-coated Si wafers, which were then exposed using a LTJ VUVES 4500 mini exposure tool at doses higher than exposure to clear (Eo) doses with 193-nm wavelength. After post-exposure bake (PEB), the substrates were developed in $2.38 \%$ tetramethylammonium hydroxide (TMAH) for 45 seconds and rinsed with deionized water. All substrates were coated and developed using a TEL Act 8 Clean Track. 


\subsection{Thickness measurements}

Monolayer thickness measurement was carried out using an M-2000® spectroscopic ellipsometer. First, the wafers were measured to obtain the native oxide thickness. Then the optical parameters of the coated films, including Cauchy coefficients, were determined. Finally, the oxide thickness and the materials' optical constants were used to build a model for determining the monolayer thickness remaining after processing on the wafer.

\subsection{Contact angle determination}

Contact angle was measured using an AST VCA Optima system with water under static conditions. The values obtained are averages of five readings.

\subsection{Atomic force microscopy (AFM)}

A Veeco NanoScope IIIa Dimension 3100 atomic force microscope (AFM) was used. Two modes of imaging were recorded, height and phase. The height mode records vertical dimensions of the topography on the substrate. The phase mode differentiates between the force interactions (attractive or repulsive) of the AFM tip and substrate surface. The differences in forces are qualitatively related to differences in composition.

\section{Results and Discussion}

\subsection{Ellipsometry results}

Silicon wafers were pre-measured to determine the native oxide on the surface. Typically the native oxide thickness was in the range 5-13 $\AA$. The amount of monolayer left after processing was measured at doses higher than the Eo to ensure complete resist exposure. In most cases exposure to clear was $>20 \mathrm{~mJ} / \mathrm{cm}^{2}$. The thicknesses of the photoresist, BARC, and combined coatings monolayers remaining after processing are shown in Table 1.

Table 1. Thickness of monolayer after processing.

\begin{tabular}{|c|c|c|c|}
\hline ArF Resist & $\begin{array}{c}\text { Resist } \\
\text { only (̊̊) }\end{array}$ & $\begin{array}{c}\text { BARC } \\
\text { only }(\AA)\end{array}$ & $\begin{array}{c}\text { Resist + } \\
\text { BARC (^̊) }\end{array}$ \\
\hline A-Vendor 1 & 3.7 & 78.7 & 63.7 \\
\hline B-Vendor 1 & 8.2 & N/A & N/A \\
\hline C-Vendor 1 & 5.1 & 6.79 & 17.9 \\
\hline D-Vendor 2 & 3.8 & 26.7 & 5.3 \\
\hline
\end{tabular}

$\mathrm{N} / \mathrm{A}=$ not available
The thickness is an average measurement from the higher three doses $\left(21-24 \mathrm{~mJ} / \mathrm{cm}^{2}\right)$. In this measurement a continuous monolayer thickness, particularly for values below $20 \AA$, is not assumed. In other words, measurement of $3.7 \AA$ for photoresist A simply implies an average in which some areas might have less and others more residue remaining.

The results show that some residue is present after both photoresist and BARC coating. When a BARC undercoating is used, the amount of residue can increase or decrease depending on the type of photoresist used. The difference can be attributed to diffusion of components (e.g., PAG) among the layers, which can contribute to or inhibit monolayer formation, although the exact nature of the diffusion components cannot be elucidated in this study. BARC shows thicker monolayer formation than photoresist. We attribute this observation to the higher affinity (polarity) of the BARC over the photoresist (as will be shown later) to the substrate.

\subsection{Contact angle results}

Water contact angle was measured under static conditions. The photoresists or BARC were coated on 4-inch wafers and baked as recommended. It was found that for photoresists the contact angle is $\sim 70^{\circ}-80^{\circ}\left(70^{\circ}\right.$ for resist $\left.\mathrm{D}\right)$, while for the BARC the angle is $43^{\circ}$. This supports the hypothesis that materials with higher polarity have more affinity to the substrate and enhance monolayer formation.

We found that increasing processing temperature enhances monolayer formation on the substrate. This behavior was true for photoresist and BARC materials. Figure 1 below shows the relationship between substrate water contact angle and BARC bake temperature (after development).

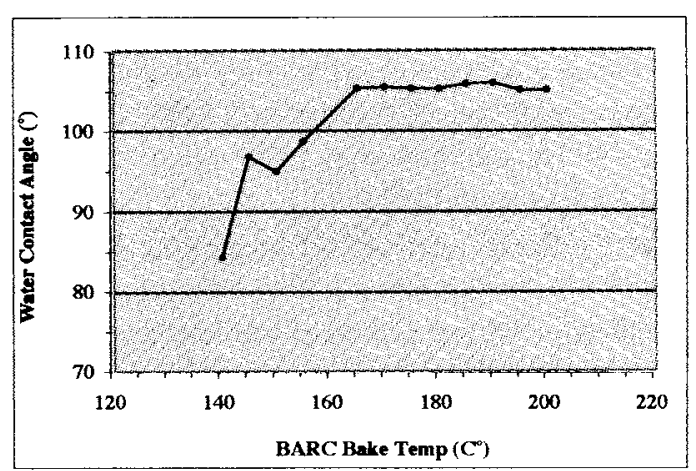

Figure 1. Substrate water contact angle vs. BARC bake (after development). 
Figure 1 results can be explained first in terms of the binding energy required for monolayer formation. The higher BARC bake temperature drives the monolayer formation forward, either by covalent bond formation or Van der Waals interactions. Figure 1 also shows that after reaching a certain temperature the contact angle remains at a steady state. At this point, the uniformity of the monolayer is homogeneous across the substrate, thus the contact angle measurement remains the same.

We can further characterize the substrate surface condition, after BARC bake and removal, by determining the total surface energy of the substrate. We used water and methylene iodide to calculate the contribution of polar and dispersive energies to the total surface energy on the substrate. The correlation between contact angle and surface energy is given by equation 1 .

Equation 1.

Surface Energy $($ dyne $/ \mathrm{cm})=\frac{1}{2}(\cos \theta+1)\left(72-\frac{\theta}{8}\right)$

Figure 2 shows that as the BARC bake temperature increases and is then removed, the substrate surface becomes less polar and has lower energy. This points to the surface becoming more organic-like and less hydrophilic due to the absence of Si-OH functionality on the surface of the substrate.

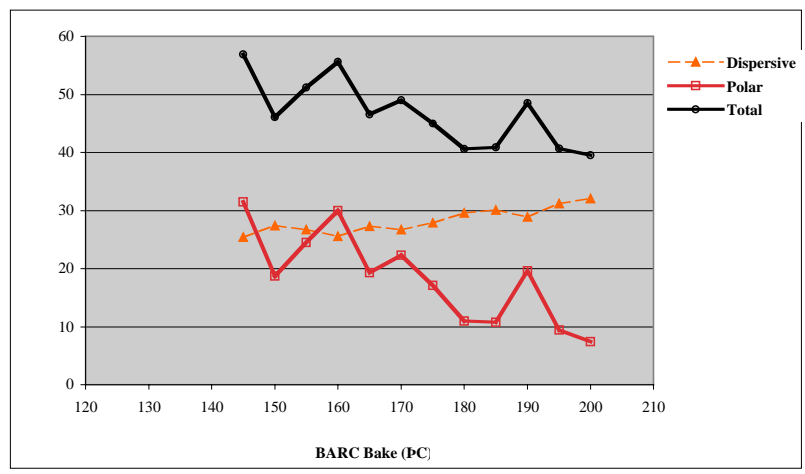

Figure 2. Dispersive, polar and total surface energy of substrate after BARC bake and removal.

\subsection{AFM results}

An AFM was used to measure surface roughness on a substrate that had been coated and processed with BARC and photoresist and a control uncoated wafer. In this experiment, silicon substrates with topography were used. The area tested was located in the open space between two line/space arrays. The AFM photographs from an uncoated (Figure 3) and previously coated (Figure 4) substrate are shown below.

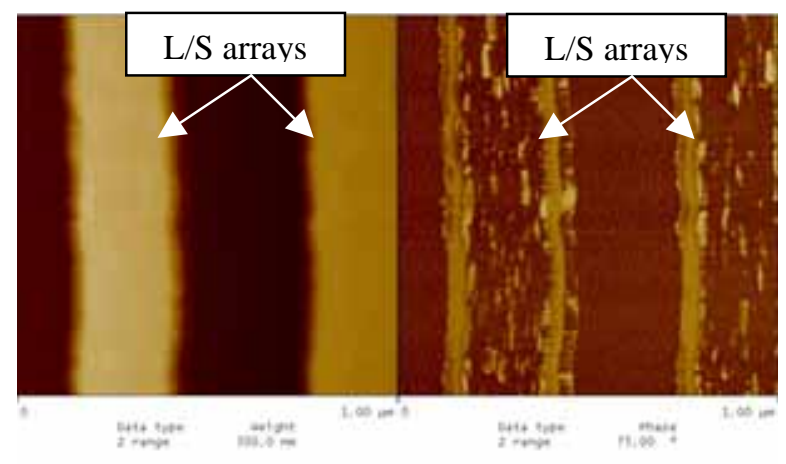

Figure 3. Height (left) and Phase (right) photograph of $1 \mu \mathrm{m}$ AFM scan of an uncoated substrate.

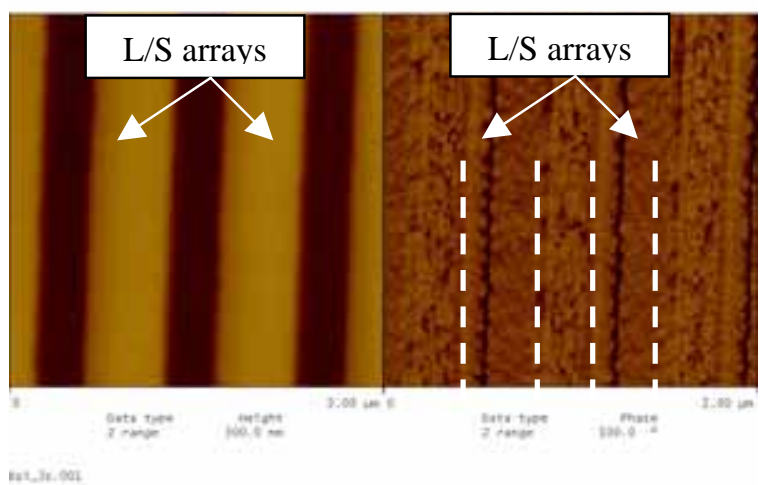

Figure 4. Height (left) and Phase (right) photograph of $2 \mu \mathrm{m}$ AFM scan of a previously coated substrate.

Comparison of Figure 4 and 5 in the Phase mode clearly show compositional differences on the substrate. The surface roughness mean square (RMS) values for uncoated, photoresist D coated and BARC coated substrates were 0.219, 0.268, and 0.656, respectively.

\subsection{Substrate dependence}

We wanted to look into the effect of substrate type on monolayer formation. In this study we studied monolayer formation of a nonphotosensitive developer-soluble KrF BARC. The monolayer thickness was measured after processing on silicon and silicon oxide substrates. Tables 2 
and 3 show the results of this study. Each substrate was measured at 4 points.

Table 2. Monolayer thickness on silicon substrate.

\begin{tabular}{|c|c|c|c|}
\hline $\begin{array}{c}\text { Silicon } \\
\text { Wafer }\end{array}$ & $\begin{array}{c}\text { Oxide } \\
\text { Pre-scan }(\AA)\end{array}$ & BARC $(\AA)$ & $\begin{array}{c}\text { Post } \\
\text { Develop }(\AA)\end{array}$ \\
\hline Average & 13.7 & 702.0 & 12.9 \\
\hline STD & 0.1 & 1.2 & 0.8 \\
\hline
\end{tabular}

Table 3. Monolayer thickness on silicon oxide substrate.

\begin{tabular}{|c|c|c|c|}
\hline $\begin{array}{c}\text { Wafer } \\
\text { with } \\
\text { SiO }_{2}\end{array}$ & $\begin{array}{c}\text { Oxide } \\
\text { Pre-scan }(\AA)\end{array}$ & BARC $(\AA)$ & $\begin{array}{c}\text { Post } \\
\text { Develop } \\
(\AA)\end{array}$ \\
\hline Average & 7539.3 & 689.8 & 2.3 \\
\hline STD & 25.3 & 0.8 & 0.2 \\
\hline
\end{tabular}

The results show that the affinity for silicon oxide is less than that for silicon substrates ( 2.3 vs $12.9 \AA$ ). Although we are trying to fully understand the reason for this observation, we believe that the more irregular surface of a thick oxide layer (stand. dev. $25.3 \AA$ ) decreases the surface area available for binding. On the silicon substrate the thin native oxide is more likely to follow crystal structure and order, thus providing for larger surface area. This explanation is illustrated in Scheme 1 below.
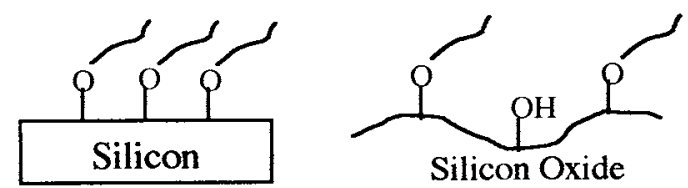

Scheme 1. Diagram illustrating surface area differences between a silicon and silicon oxide substrates.

\subsection{Comparison between methods}

The relationship between direct thickness measurement and water contact angle is show in Figure 5. Figure 6 shows the correlation RMS taken by AFM and thickness measurement.

Figure 5 shows that there is a direct relationship between contact angle and monolayer thickness until a certain thickness is achieved. After about 10 $\AA$ of measurable thickness, there is no change in contact angle. When the monolayer is thinly dispersed across the wafer, the water contact angle measurement gives an average of response of the water droplet on the surface of hydrophobic and hydrophilic areas. As the monolayer thickness becomes more uniform, the water droplet is only in contact with the monolayer film and thus gives a steady contact angle value. At this point the monolayer thickness can increase but it had no impact on the contact angle. Unlike the water contact angle test, the RMS obtained from AFM measurements does follow thickness measurements.

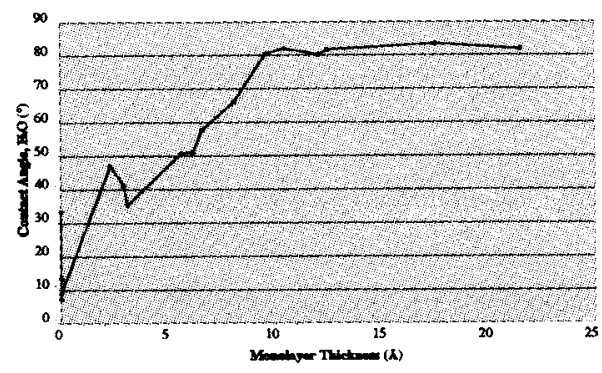

Figure 5. Water contact angle vs. monolayer thickness.

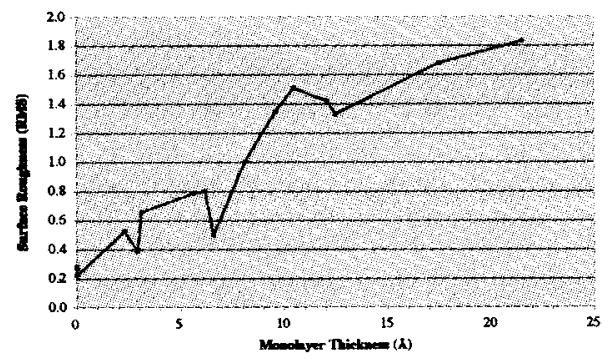

Figure 6. RMS vs. monolayer thickness.

\subsection{Conclusions}

The surfaces of silicon and silicon oxide substrates were analyzed after being coated and processed with photoresist and/or BARCs. AFM, contact angle, and ellipsometry studies show that the substrate has monolayer level material remaining. These low-level residues do not appear to be problematic in subsequent processes such as substrate etch, implant, or others. From all three methods, direct thickness measurement using ellipsometry seem to be more reliable at measuring monolayer level residue on semiconductor substrates. We will further investigate the effect of monolayer residues on semiconductor substrates.

\section{Acknowledgments}

The authors would like to acknowledge the experimental contributions from Carlton Washburn 
and Alice Guerrero from Brewer Science, and Rishi Patel from Missouri State University.

\section{References}

1. R. P. Wool, Polymer Interfaces: Structure and
Strength, Hanser Publishers, New York, (1995).

2. H. Jo and F. Blum, Chem. Mater., 11 (1999), 2548.

3. D. Ahn, K. R. Shull, Langmuir, 14 (1998) 3646. 